\title{
Temporal Evolution of Plasma From a Highly Ionized Helium Capillary Discharge
}

\author{
MAYO VILLAGRAN AND JORGE J. ROCCA, MEMBER, IEEE
}

\begin{abstract}
Emission spectroscopy was used to study the temporal evolution of a highly ionized helium plasma generated in a 1-mm-diameter capillary by a fast (90-ns full width at half-maximum (FWHM)) discharge pulse. The time dependence of the plasma density was measured from the Stark broadened line profile of the HeII 468.5-nm transition. A 1.2-kA discharge current pulse was observed to create a plasma density of $8 \times 10^{16} \mathrm{~cm}^{-3}$ in 1 torr of helium. The maximum intensity of Hell transitions occurs in the afterglow of the discharge pulse, following the collisional recombination of totally stripped ions with plasma electrons when the plasma cools. The study is of interest in relation to the possibility of obtaining amplification in the $164.0-\mathrm{nm}$ line of HeII in a capillary discharge.
\end{abstract}

\section{INTRODUCTION}

$\mathrm{F}^{\mathrm{s}}$ AST CAPILLARY discharges can generate highly onized plasmas with a large length-to-diameter ratio [1]-[4]. Our interest in these plasmas is related to their potential use in the generation of vacuum ultraviolet and extreme ultraviolet laser radiation from population inversions created by electron impact excitation either during the current pulse or following collisional electron-ion recombination in the afterglow. Longitudinal discharges in tubes with 4-7 mm bore and peak current densities of the order $10000 \mathrm{~A} / \mathrm{cm}^{2}$ have been used previously to produce laser action by electron impact ionization at wavelengths as short as $175 \mathrm{~mm} \mathrm{[5]-[7].} \mathrm{Higher} \mathrm{current} \mathrm{den-}$ sities in smaller diameter tubes might allow the generation of laser radiation at shorter wavelengths.

Rapid cooling of a capillary plasma at the termination of the current pulse would result in the recombination of multiply charged ions, which by preferentially populating the highest energy levels of ions of lower charge could lead to population inversion and gain at short wavelength [7], [8]. Population inversion between the levels of the 53 and 4-3 transitions of HeII has been observed following plasma recombination in a flowing arc jet [9]. However the low density $\left(8 \times 10^{13} \mathrm{~cm}^{-3}\right)$ of this plasma limited the single-pass gain for these lines to less than 1.03 . The rapid recombination of a more dense plasma is required to generate the larger population inversion necessary to

Manuscript received November 7, 1989; revised May 31, 1990. This work was supported in part by the National Science Foundation under grant ECS-8606226, in part by a National Science Foundation Presidential Young Investigator Award, and in part by a postdoctoral fellowship provided by the Organization of American States.

The authors are with the Electrical Engineering Department, Colorado State University, Fort Collins, CO 80523.

IEEE Log Number 9038310 obtain laser action at $164 \mathrm{~nm}$ in the 3-2 transition of HeII. Capillary discharges have potential advantages for the generation of the plasma condition necessary for amplification. In a capillary structure, a high current pulse can be used to create a highly ionized plasma with a long optical path. Electron heat conduction to the capillary walls and plasma radiation can provide rapid plasma cooling at the termination of the current pulse, thereby creating the conditions for rapid recombination.

Herein we discuss the generation of a highly ionized helium capillary plasma, and the study of its temporal evolution. A $30-\mathrm{cm}-$ long and $1-\mathrm{mm}$-diameter helium plasma was created with well-terminated kiloamp current pulses of $90 \mathrm{~ns}$ full width at half-maximum (FWHM). Emission spectroscopy was used to study the recombination of totally stripped ions into hydrogenic helium ions, and to measure the evolution of the plasma density from the Stark broadening of HeII transitions. The results are discussed in relation to the possibility of obtaining laser action in the 164-nm line of hydrogenic helium.

\section{Generation of the Capillary Plasma}

The discharge setup used in the experiment is schematically illustrated in Fig. 1. A capillary helium plasma is created in a precision-bored quartz tube of $1 \mathrm{~mm}$ in diameter and $30 \mathrm{~cm}$ in length by discharging a $4.4-\mathrm{nF}$ capacitor through a low-inductance circuit consisting of a triggered spark gap in series between the capacitor and the capillary tube. The capacitor is composed of a ring of four 1.1-nF ceramics capacitors that surrounds the cylindrical cathode electrode, and like the spark gap, is enclosed in a Plexiglas container filled with transformer oil. The current return from the anode electrode, placed at the opposite extreme of the capillary, occurs through a copper tube, which is coaxial and closely spaced to the capillary tube in a configuration that minimizes the circuit inductance. The total circuit inductance was measured to be 0.5 $\mathrm{uH}$. The copper tube has a central slot $20 \mathrm{~mm}$ long and 3 mm wide to allow the spectroscopic analysis of the radially emitted radiation.

The discharge current is measured with a current transformer coil that surrounds the discharge tube and is recorded with a waveform digitizer. Previous to the discharge, the device is evacuated through both electrodes with a turbomolecular pump. Then the turbomolecular pump is turned off and helium is admitted through both electrodes while the discharge setup is evacuated from the 


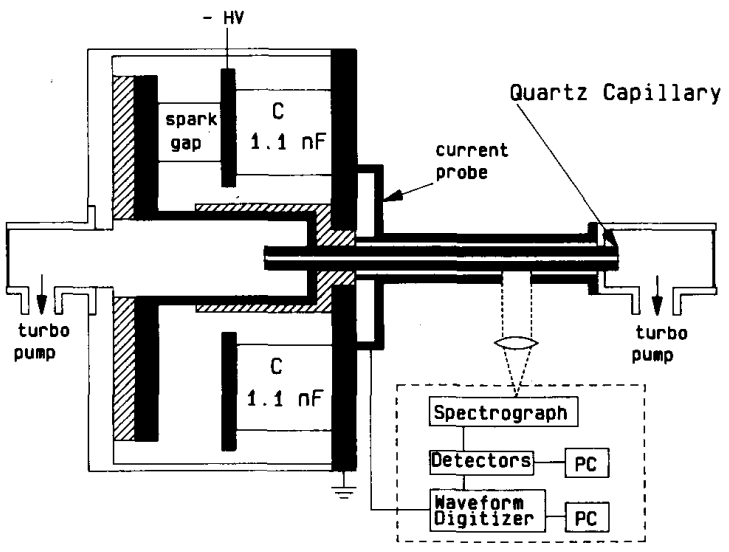

Fig. 1. Schematic representation of capillary discharge setup.

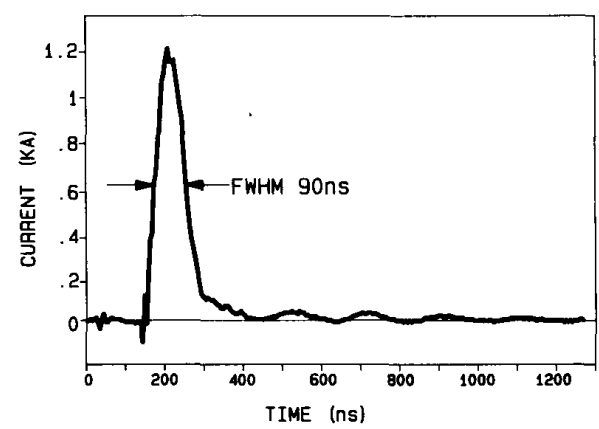

Fig. 2. Current pulse corresponding to 20-kV 1-torr helium discharge excited by $4.4-\mathrm{nF}$ capacitor. Diameter of capillary is $1 \mathrm{~mm}$ and length is $30 \mathrm{~cm}$.

anode side with a rotary pump. The pressure on the cathode side is maintained at a slightly higher value to establish a small continuous gas flow through the capillary, facilitating the flushing out of gaseous impurities. In the experiments discussed herein, the discharge was operated at a frequency of $1 \mathrm{~Hz}$.

Fig. 2 shows the discharge current pulse obtained when the capacitor is charged to $20 \mathrm{kV}$ and the pressure in the capillary tube is 1 torr. The current peak reaches $1.2 \mathrm{kA}$, corresponding to a current density of $1.510^{5} \mathrm{~A} / \mathrm{cm}^{2}$, and it has a FWHM of nearly $90 \mathrm{~ns}$. The length of the discharge tube, $30 \mathrm{~cm}$, was selected to obtain a sufficiently large plasma resistance such that the current pulse is well terminated, with negligible current after the first half-circle.

\section{Spectral Emission of the Helium Capillary Plasma}

Fig. 3 shows time-integrated spectra of the radiation emitted in the $200-600 \mathrm{~nm}$ spectral region by a 1-torr helium capillary discharge excited by a $20-\mathrm{kV}$ pulse. The spectra were obtained collecting and focusing the radial spontaneous emission from the discharge with quartz lenses into the slit of a $30-\mathrm{cm}$ focal-length spectrograph

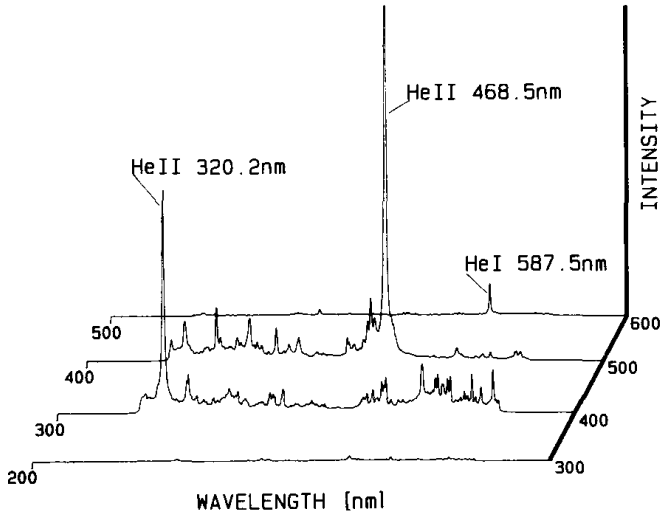

Fig. 3. Time-integrated spectra (200-600 nm) of radial emission of 1-torr 1-mm-diameter helium capillary discharge excited by discharge current pulse shown in Fig. 1.

provided with a 600 lines $/ \mathrm{mm}$ grating. The detection was accomplished using an intensified diode array detector. The 468.5-nm and 320.3-nm Hell lines, corresponding to the 5-3 and 4-3 transitions, dominate the spectrum. The only HeI line observed is the $587.5-\mathrm{nm}$ transition with an intensity one order of magnitude smaller than the ionic lines. This observation indicates, as later corroborated by electron density measurements, that the plasma is highly ionized. The lines with smaller intensity in the same spectrum were identified to be silicon and oxygen lines associated with material liberated from the capillary wall by the discharge pulse.

Considerable information on the plasma evolution can be obtained by analyzing the temporal dependence of the emission from these ionic spectral lines. Of particular interest are the 468.5 and 320.3-nm lines, which, as illustrated in the simplified energy level diagram of Fig. 4, populate the $n=3$ level of HelI by radiative and collisional cascade. This is the upper level of the potential 164.0-nm laser line. The temporal dependence of Hell line emission was studied replacing the array detector with a photomultiplier tube. The detection system was placed in a Faraday enclosure to shield it from the electromagnetic noise generated by the fast discharge pulses.

Fig. 5 shows the temporal evolution of the $468.5-\mathrm{nm}$ line intensity and the corresponding current pulses for $10-, 15-$, and $20-\mathrm{kV}$ excitation of a 1 -torr discharge. The relative temporal position of the emitted radiation with respect to the current pulse was corrected taking into account the delays in the cables and the transit time of the photoelectrons in the photomultiplier. For $10-\mathrm{kV}$ excitation, the emission closely follows the current pulse and there is little radiation after the termination of the current pulse. This radiation pulse corresponds to excitation by electron impact and to an insignificant amount of recombination of totally stripped ions into HeII in the afterglow of the discharge. When the excitation voltage is increased to $15 \mathrm{kV}$, the intensity of the $468.5-\mathrm{nm}$ emission during the time interval of the current pulse is observed to slightly increase, while a large increase in the afterglow intensity 


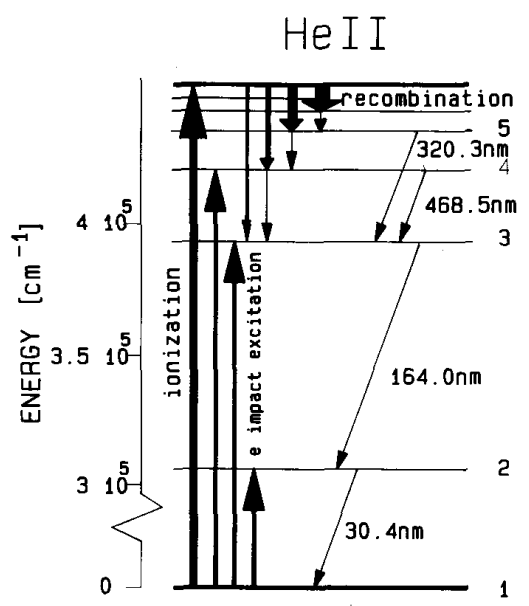

Fig. 4. Simplified atomic energy level diagram of ionized helium including relevant population processes.

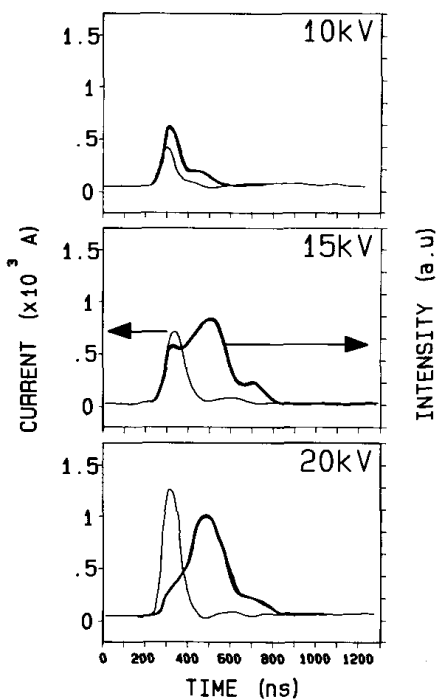

Fig. 5. Time evolution of current pulse and corresponding HeII 468.5-nm radial emission for different discharge voltages for 1-torr He discharge.

is observed. The peak of the 468.5-nm line intensity in this case occurs $200 \mathrm{~ns}$ after the peak of the current pulse, indicating strong excitation on the $n=4$ level of HeII following the recombination of totally stripped ions. Further increase of the excitation voltage to $20 \mathrm{kV}$ causes a slight additional increase of the afterglow intensity while the emission during the current pulse, which now peaks at $1.2 \mathrm{kA}$, is observed to be significantly reduced. This behavior suggests that the plasma reaches a very high degree of ionization during the current pulse, as confirmed by plasma density measurements.

\section{Temporal Evolution of the Plasma Density}

We recorded the temporal variation of the Stark broadened profile of the 468.5-nm transition of Hell to determine the evolution of the plasma density. The large

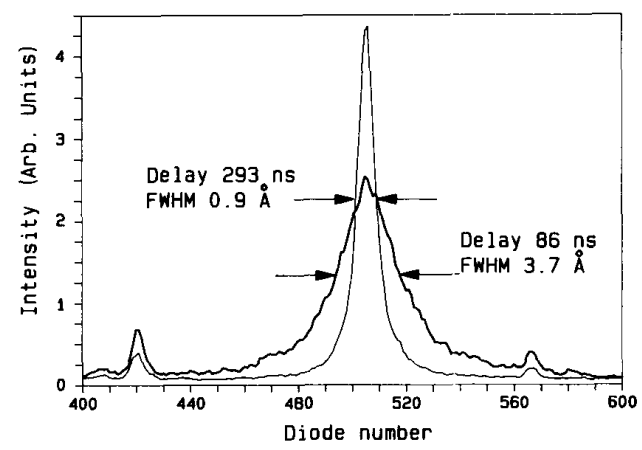

Fig. 6. Lineshape of HeII 468.5-nm line taken $86 \mathrm{~ns}$ and $293 \mathrm{~ns}$ after initiation of discharge current pulse. Helium pressure was 1 torr and excitation voltage was $20 \mathrm{kV}$.

Stark broadening of this line provides a nonintrusive method of determining the plasma density, which is relatively insensitive to the value of the electron temperature [10]. To record the line profiles, we used a $0.75-\mathrm{m}$ focal length spectrograph with a 1800 lines $/ \mathrm{mm}$ grating. Time resolution was obtained gating the intensifier of the array detector with an 18-ns-wide voltage pulse. The signal from the current monitor transformer coil was used to trigger the gating pulse to the multichannel plate intensifier through a variable time delay circuit.

Fig. 6 displays the line profile of the HeII 4-3 line at two different times during the plasma evolution. The broader line profile corresponds to the emission recorded shortly after the peak of a 1.2-kA current pulse of a 1-torr discharge. The narrower spectrum corresponds to the emission of the same transition at 293 ns after the initiation of the current pulse, which is coincident with the portion of the afterglow occurring $117 \mathrm{~ns}$ after the termination of the current pulse. The large difference in the linewidths is due mainly to Stark broadening and can be used to determine the electron density. Corrections were made taking into account the contributions of Doppler and instrumental broadening. The instrumental linewidth was determined to be $0.5 \mathrm{~A}$ using low-pressure spectral lamps.

The majority of the measurements were obtained collecting the radially emitted light without discriminating between the contributions of different radial zones. In a few cases, spatially resolved data were obtained by displacing two parallel slits across the discharge diameter. The spatial resolution of the arrangement was $0.1 \mathrm{~mm}$. In these cases, the electron density was calculated by Abel inversion, and the density in the discharge axis was found to be in very good agreement with that obtained from the spatially integrated measurements. At the time of the maximum emission from the recombination excited lines, the plasma density distribution was measured to be relatively flat across the majority of the discharge tube diameter, as a result of plasma diffusion.

Fig. 7 shows the measured evolution of the plasma density for helium discharges at pressures of 1,2 , and 3 torr excited by $20-\mathrm{kV}$ pulse. The plasma density in the 1 -torr discharge was measured to reach $8 \times 10^{16} \mathrm{~cm}^{-3}$. This 


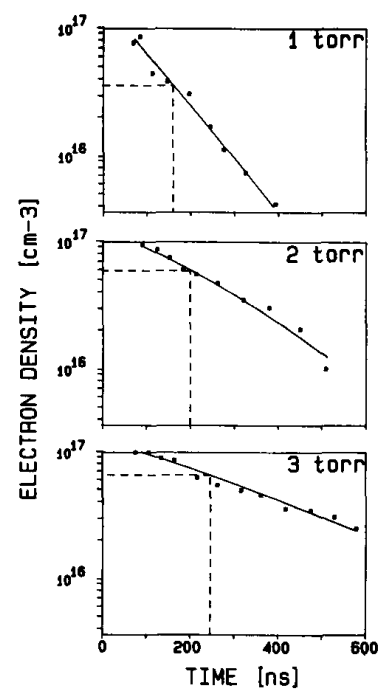

Fig. 7. Temporal evolution of plasma density for 1,2 , and 3 torr of He in 1-mm-diameter capillary discharge excited by $20 \mathrm{kV}$ pulse. Times are measured from beginning of current pulse. Dashed lines indicate plasma density at time of maximum recombination excited emission of Hell lines.

density is slightly above the value corresponding to complete ionization of 1-torr helium column at room temperature, $7.2 \times 10^{16} \mathrm{~cm}^{-3}$, and could result from a slight self-constriction of the plasma column under the influence of the self-generated magnetic field. The peak value of the plasma density increases up to $1 \times 10^{17} \mathrm{~cm}^{-3}$ when the pressure is increased from 1 to 2 torr, as more helium atoms are available to be ionized. However, notice that the degree of ionization of the plasma decreases as the helium pressure is increased. The rate of the plasma density decay is also observed to decrease as the pressure is increased, as a consequence of reduced particle diffusion. The dashed lines in Fig. 7 identify the plasma density corresponding to the time of the recombination peak. By the time corresponding to the maximum emission from the 468.5-nm line in the 1-torr discharge, occurring approximately $110 \mathrm{~ns}$ after the peak of the current pulse, the plasma density is observed to decrease to approximately $3.5 \times 10^{16} \mathrm{~cm}^{-3}$ as a result of charged particle diffusion and recombination.

Assuming the plasma is completely ionized, the electron temperature can be estimated from the plasma resistivity [11]. Using the plasma resistance value of $10 \Omega \mathrm{ob}-$ tained by fitting the measured shape of the current pulse with that predicted from an LRC circuit model, a value of $16 \mathrm{eV}$ is obtained. However, this temperature estimation cannot be considered accurate because it includes the simplifications of assuming a constant plasma resistance and of considering that the current flow covers the entire capillary tube diameter. Computing the ionization rate, it can be shown that the electron temperature required to achieve the observed near-complete ionization of the helium gas in the time interval in which the current rises to its maximum value ( $50 \mathrm{~ns}$ ) must be at least $20 \mathrm{eV}$.
In the next section, we discuss these results in relation to the possibility of generating laser radiation by electron-ion recombination at $164.0 \mathrm{~nm}$ from the 3-2 transition of HeII.

\section{Discussion}

To determine the necessary plasma conditions for amplification at $164.0 \mathrm{~nm}$, we calculated the small signal gain of this transition as a function of the plasma density and the electron temperature using a collisional-radiative model. The gain was computed after determining the population densities by solving a set of rate equations, including all the excited states of hydrogenic helium with principal quantum numbers up to 12 . The relative density of ground-state singly and doubly ionized helium ions, the electron density, and the electron temperature are input parameters for the calculation.

The processes considered to determine the populations of the different states are electron impact ionization, collisional excitation and de-excitation, three-body and radiative recombination, and radiative decay due to spontaneous emission. The ion and the electron temperatures were assumed to be equal, and the calculations were done for an optically thin plasma. The linewidths were calculated taking into account both Doppler and ion quasi-static broadening mechanisms. The collisional rate constants were taken from the literature [12], [13], and rates for the reverse processes were calculated by using the principle of detailed balance. For electron impact excitation, the rates derived by Seaton [13] were used, with a Gaunt factor as calculated by Van Regemorter [14] but scaled to a value of 0.4 for the electron energy range where $(\Delta E / k T)$ $>1$, as suggested by Bates et al. [15].

Fig. 8 illustrates the predicted gain for plasma densities of $4 \times 10^{15}, 8 \times 10^{15}, 1 \times 10^{16}$, and $1.2 \times 10^{16} \mathrm{~cm}^{-3}$ for the case in which the ratio of totally ionized atoms to ground-state singly ionized atoms is 0.5 . The plot shows that to achieve a gain greater than $0.01 \mathrm{~cm}^{-1}-\mathrm{a}$ value sufficiently large to produce laser oscillation when a good optical cavity is provided-plasma temperatures of less than $1.5 \mathrm{eV}$ are required at the time the recombining plasma reaches the specified ion distribution. For the case in which the density of totally ionized atoms equals the density of ground-state singly ionized atoms at the time the plasma reaches the specified temperature, the gain values are computed to increase by approximately a factor 1.3 with respect to those of Fig. 8, while for the case of a ratio of $\mathrm{He}^{++}$to $\mathrm{He}^{+}$equal to 0.2 , the gain curves are similar to those in Fig. 8 but with values lowered by a factor between 0.5 and 0.6 . The calculation shows that no inversion is obtained at plasma densities greater than 1.2 $\times 10^{16} \mathrm{~cm}^{-3}$, due to electron impact de-excitation of the upper level. Also, when re-absorption of radiation is taken into account, trapping of the radiation originated in the $n=2$ level sets a limit for the density of ground-state singly charged ions, and thereby determines a maximum plasma density at which an inversion can be obtained [16]. 


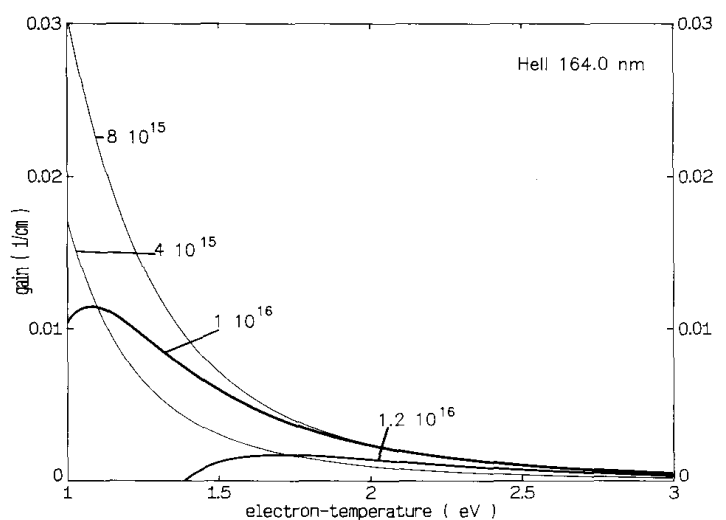

Fig. 8. Calculated gain for HeII $164.0-\mathrm{nm}$ line as function of plasma temperature and density. Ratio of completely ionized atoms to hydrogenic ions assumed to be equal to 0.5 . Electron densities in units of $\mathrm{cm}^{-3}$.

Since for the discharge conditions discussed in Section II the plasma density at the time of maximum recombination exceeds the maximum value set by electron impact de-excitation, we made attempts to reduce the plasma density by decreasing the helium filling pressure below 1 torr. Unfortunately, that led to plasma nonuniformities. Visual observation and time-integrated photographs of the discharge showed that the plasma changes from an axially very uniform plasma into a nonuniform plasma presenting multiple bright spots. While we have not studied this phenomenon, it is apparent that it occurs when the plasma temperature is high and the plasma reaches near-complete ionization, and it might be associated with a magnetohydrodynamic instability, such as the $m=1$ helical instability commonly observed in gas embedded pinches [17]. Also, our measurements indicate that when the filling gas pressure is decreased to 0.5 torr, the plasma emission due to wall impurities becomes important.

In summary, a nearly completely ionized helium capillary plasma with a density of $8 \times 10^{16} \mathrm{~cm}^{-3}$ was created by a 1.2-kA discharge current pulse. The maximum intensity of the emission from hydrogenic helium lines occurs in the afterglow of the discharge; it is caused by excitation following the collisional recombination of totally stripped ions as the plasma cools. For the conditions at which a uniform plasma column was created, the value of the plasma density at the time of maximum recombination was determined to be above the limit imposed by collisional de-excitation for the generation of a population inversion in the 3-2 transition of HeII.

\section{ACKNOWLEDGMENT}

The authors wish to thank D. Beethe for implementing the computer code used in the gain calculations, and $\mathrm{M}$. Marconi for the critical reading of the manuscript.

\section{REFERENCES}

[1] H. Conrads, "Die erzeugung von seriengrenzkontinua mit hilfe von gleitfunken zur absolutmessung im vakuum ultraviolet,"' $Z$. fur Physik, vol. 220, pp. 444-455, 1967.
[2] P. Bogen, H. Conrads, G. Gatti, and W. Kohlhaas, "Continuum radiation source of high intensity," J. Opt. Soc. Amer., vol. 58, pp. 203-206, 1968.

[3] R. A. McCorkle, "The high power sliding-spark capillary discharge in vacuum: Variations and applications," Appl. Phys. A, vol. 26, pp. 261-270, 1981

[4] M. C. Marconi and J. J. Rocca, "Time resolved extreme ultraviolet emission from a highly ionized capillary discharges," Appl. Phys. Lett., vol. 54, pp. 2180-2182, May 1989.

[5] J. Marling and D. B. Lang, "Vacuum ultraviolet lasing from highly ionized noble gas," Appl. Phys. Lett., vol. 31. pp. 181-184, 1977.

[6] G. A. Massey, B. P. Plummer, and J. C. Johnson, "A high repetition rate ion laser spanning the $195-225 \mathrm{~nm}$ spectral region," IEEE $J$. Quant. Elect., vol. QE-14, pp. 673-679, 1978.

[7] J. J. Rocca, D. C. Beethe, and M. C. Marconi, "Proposal for soft$\mathrm{X}$-ray and XUV laser in capillary discharges," Opt. Lett., vol. 13, pp. 565-567, July 1988 .

[8] J. J. Rocca, M. C. Marconi, D. Beethe, and M. Villagran Muniz, "Prospects of XUV recombination lasers in capillary discharges," in Proc. Int. Conf. on Lasers'88, R. C. Sze and F. J. Duarte, Eds. McLean, VA: STS Press, 1989 , pp. 278-285.

[9] T. Hara, T. Ohgo, M. Hamagaki, and T. Dote, "Quasi-steady population inversion of $\mathrm{He}^{+}$in a freely expanding plasma, "Japan. $J$. App. Phys., vol. 21, pp. 461-463, 1982.

[10] H. R. Griem, Plasma Spectroscopy. New York: McGraw-Hill, 1964.

[11] L. Spitzer Jr., Physics of Fully lonized Gases. New York: Wiley \& Sons, 1962.

[12] R. W. McWhirter, Plasma Diagnostic Techniques, R. H. Huddlestone and S. L. Leonard, Eds. New York: Academic Press, 1965.

[13] M. J. Seaton, Atomic and Molecular Processes, D. R. Bates, Ed. New York: Academic Press, 1962, p. 375.

[14] H. Van Regemorter, "Rate of collisional excitation in stellar atmospheres," Astrophys. J., vol. 130, p. 912, 1962

[15] D. R. Bates, F. Kingston, and R. McWhirter, "Recombination between electrons and atomic ions I. Optically thin plasmas," Proc. Roy. Soc. A, vol. 267, pp. 297-312, May 1962.

[16] O. R. Wood II and J. W. Silfvast, "Electron density and energy output limits of plasma-recombination lasers," Appl. Phys. Lett., vol. 41, pp. 121-123, July 1982 .

[17] J. E. Hammel, D. W. Scudder, and J. S. Shalachter, "Recent results on dense Z pinches,' Nucl. Insirum. Methods Phys. Res., vol. 207, nos. 1-2, pp. 161-168, Mar, 1983

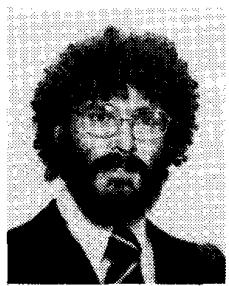

Mayo Villagran was born in Montevideo, Uruguay, on May 2, 1954. He received the Diploma in Physics and the Doctor in Physics degrees from the University of La Plata, Argentina, in 1978 and 1986, respectively.

From 1986 to 1988 he was at Colorado State University on a fellowship of the Organization of America States. Presently, he is working at the Universidad Autonoma de Mexico, developing medical applications of lasers.

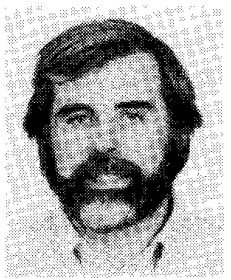

Jorge J. Rocca (S'80-M'83) received the Diploma in Physics from the University of Rosario, Argentina, in 1978, and the Ph.D. degree from Colorado State University, Fort Collins, in 1983. $\mathrm{He}$ is currently an Associate Professor in the Department of Electrical Engineering at Colorado State University. His broad research interests include the development of new short-wavelength lasers and the physics of discharges and plasmas. His work has included the development of ion lasers excited by electron beams, the generation of intense electron beams from glow discharges, the demonstration of recombination lasing in negative glow plasmas, and the study of dense capillary discharges, which he has proposed as a possible source of soft X-ray and extreme ultraviolet recombination laser radiation

Dr. Rocca received a National Science Foundation Presidential Young Investigator Award during 1985-1990, and he is also a recipient of the Halliburton Foundation Award. He is a member of the Optical Society of America. 